\title{
Viscoelasticity of Pediatric Blood and its Implications for the Testing of a Pulsatile Pediatric Blood Pump
}

\author{
Jennifer A. Long, ${ }^{*}$ Akif Ündar, ${ }^{*}+$ Keefe B. Manning, ${ }^{*}$ and Steven Deutsch*
}

Red blood cell hematocrit, aggregation and deformability, and plasma protein concentration influence the viscosity and elasticity of whole blood. These parameters affect the flow properties, especially at low shear rates $\left(<50 \mathrm{~s}^{-1}\right)$. In particular, we have previously shown that the viscoelasticity of fluid affects the inlet filling characteristics and regions of flow separation in small pulsatile blood pumps. Although the viscosity of pediatric blood has been thoroughly studied, its elasticity has not been previously measured. Here we present the viscosity and elasticity of pediatric blood against shear rate for hematocrits from 19-56, measured using an oscillatory rheometer. There is little effect of patient age on blood viscoelasticity. A statistical analysis showed that when compared at constant hematocrit, blood from adult and pediatric patients had similar viscoelastic properties. We present blood analog solutions, as a function of hematocrit, constructed on the basis of the pediatric measurements. Flow field results for viscoelastic analogs of 20, 40 and $60 \%$ hematocrit and a Newtonian analog will be compared in the initial, in vitro testing of the Penn State pediatric blood pump, to determine the importance of incorporating a viscoelastic analog into the desigh interaction. ASAIO Journal 2005; 51:563-566.

B ecause of improvement in surgical techniques and an increase in pediatric patients awaiting heart transplants, mechanical cardiac support has become a more frequent treatment option. ${ }^{1}$ Initial attempts by Penn State to make a 15 -cc version of their 70-cc ventricular assist device (VAD) were not successful because of thrombus formation in animal models. ${ }^{2}$ Areas of low shear stress lead to insufficient wall washing, allowing blood clots to develop. Previous work by Bachmann et al. ${ }^{2}$ demonstrated the differences in Newtonian and nonNewtonian blood analogs when testing a pediatric VAD. When conducting in vitro testing to measure the fluid mechanics in the pediatric VAD, it is important to use a fluid that mimics properties of pediatric blood.

Blood is a viscoelastic fluid that behaves as a Newtonian fluid at shear rates above $500 \mathrm{~s}^{-1}$. At shear rates lower than 50

From the *Department of Bioengineering, The Pennsylvania State University, University Park, PA; and +Department of Pediatrics, Penn State College of Medicine, Hershey, PA.

Submitted for consideration June 2005, and accepted in revised form July 2005.

Presented in part at the First International Conference on Pediatric Mechanical Circulatory Support Systems and Pediatric Cardiopulmonary Perfusion, May 19-22, Hershey, PA, USA.

Reprint Requests: Steven Deutsch, PhD, The Pennsylvania State University, Department of Bioengineering, 205 Hallowell Building, University Park, PA, 16802; e-mail: sd1 @wt.arl.psu.edu

DOI: 10.1097/01.mat.0000180353.12963.f2 $\mathrm{s}^{-1}$, the viscosity of blood increases exponentially due to the formation of large aggregates of erythrocytes. As shear rate increases, the erythrocytes are dispersed and aligned in the direction of flow. ${ }^{3}$

Newtonian blood analogs are often used for in vitro experiments, but it has been shown that these fluids produce higher values of wall shear stress and Reynolds stresses than nonNewtonian fluids. ${ }^{2}$ The lower stresses seen in viscoelastic fluids are more conducive to thrombus formation. To determine appropriate fluids to be used for testing a pediatric VAD, the viscoelasticity of pediatric blood must first be determined. The viscoelasticity of blood is strongly dependent on hematocrit, with weaker dependence on plasma viscosity, platelets, and plasma proteins. ${ }^{4}$ This dependence should be taken into account when establishing normal values $3,5,6$ in order to develop blood analogs and to compare results.

\section{Measurement Principles}

To determine the viscoelasticity, the fluid is oscillated at a known frequency in a rigid cylindrical tube. The flow rate and pressure are measured throughout the cycle, and from these the shear stress and shear rate can be calculated. For a Newtonian fluid, the shear stress and shear rate are in phase, but for a non-Newtonian fluid, the phase shift between the two leads to a complex viscosity:

$$
\eta=\eta^{\prime}-\mathrm{i} \eta^{\prime \prime}
$$

where $\eta^{\prime}$ is the viscosity and $\eta^{\prime \prime}$ is the elasticity. They are related to dissipated and recovered energy, respectively. The two components of complex viscosity allow the elastic stress to be separated from the viscous stress. The yielding of red blood cell aggregates becomes evident when looking at the relationship between elastic stress and strain. The yield stress is related to the point when blood reaches a superfluid state, and loses its ability to store energy. ${ }^{7}$ The measurement of the viscosity, elasticity, yield stress, and relaxation time as opposed to viscosity only, has the advantage of obtaining information about aggregability and deformability of cells.

Constructing a complex viscosity does not require a model to be assumed for blood viscoelasticity. From these components, other viscoelastic parameters can be determined by introducing a rheological model. By way of example, we use the Maxwell model here, which consists of an idealized spring and dashpot in series. ${ }^{8}$ Using this model, the strain rate $(\dot{\gamma})$ can be written in terms of shear stress $(\tau)$ and shear rate $(\dot{\tau})$ :

$$
\dot{\gamma}=\frac{\dot{\tau}}{G}+\frac{\tau}{\eta}
$$

Where $G$ is the shear modulus of the spring and $\eta$ is the viscosity of the fluid in the dashpot. By imposing an instanta- 

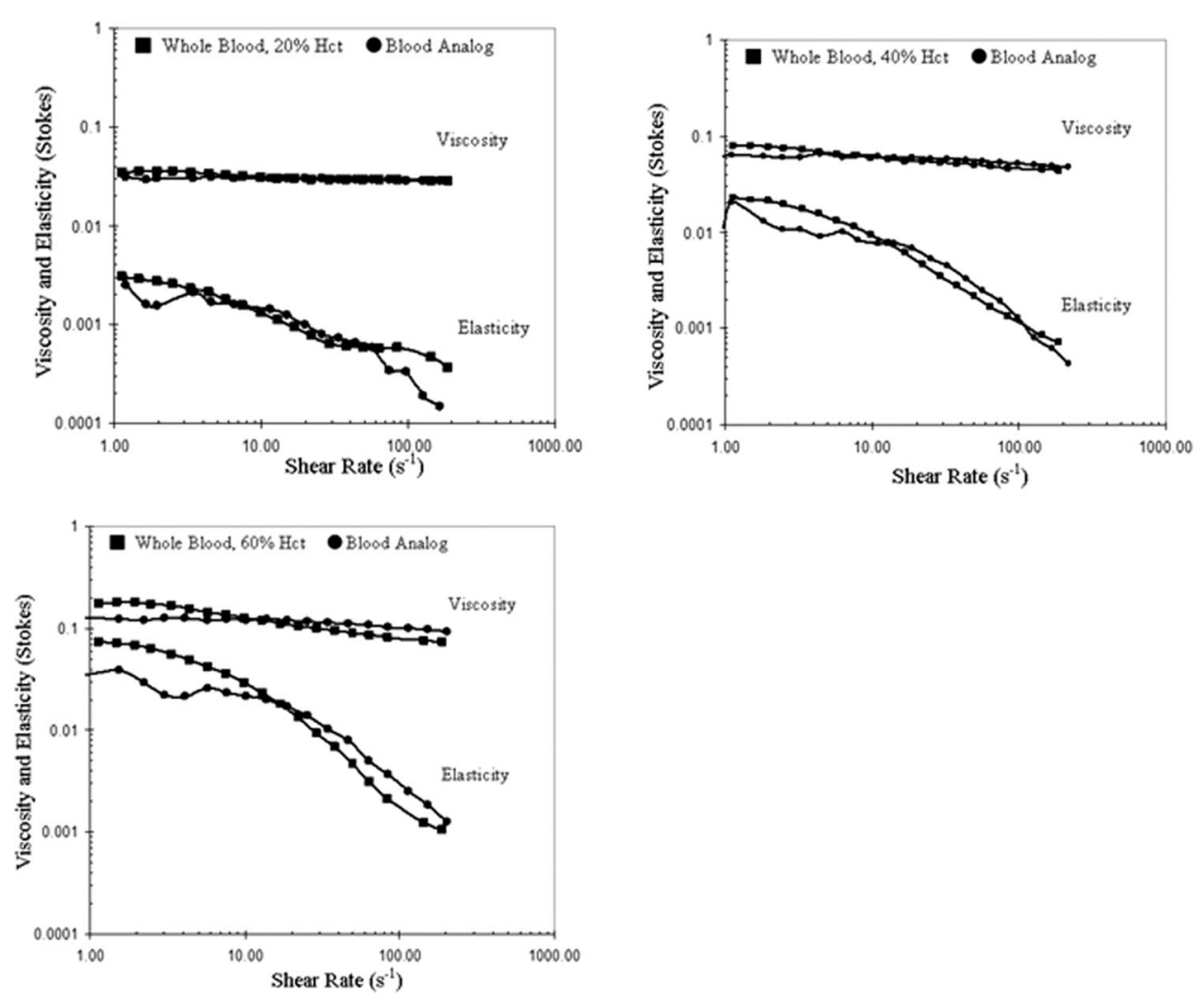

Figure 1. Viscosity and elasticity of pediatric blood and blood analogs at $20 \%, 40 \%$, and $60 \%$ hematocrit.

neous strain to the model, the shear stress as a function of time ( $t)$ can be written as:

$$
\tau(t)=\tau_{0} e^{-t / \lambda}
$$

The initial stress response $\left(\tau_{0}\right)$ decays exponentially with time, with the time constant of the material $(\lambda)$ referred to as the relaxation time. The relaxation time can be calculated in terms of the complex viscosity and frequency of the oscillations $(f)$ :

$$
\lambda=\frac{\eta^{\prime \prime}}{2 \pi \int \eta^{\prime}}
$$

For blood, $\lambda$ relates to the speed at which the cells deform and reorient themselves during flow. ${ }^{9}$

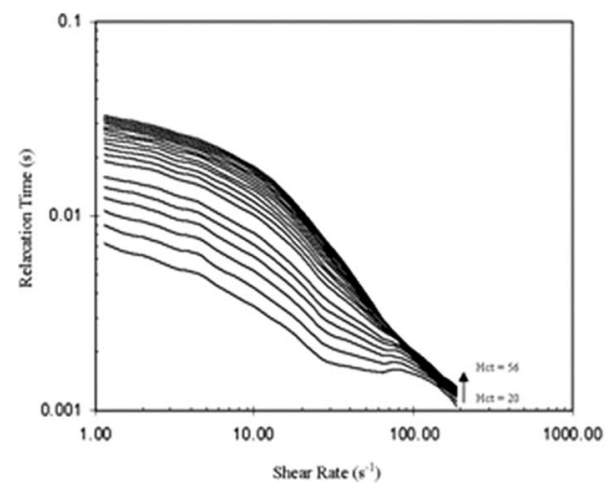

\section{Materials and Methods}

After Institutional Review Board approval, 21 pediatric cardiac patients were enrolled in this study. Arterial blood samples were taken. The test was completed in 3 minutes and required $1.0 \mathrm{ml}$ anticoagulated blood. Blood samples were tested with the Vilastic-3 Viscoelasticity Analyzer (Vilastic Scientific, Inc., Austin, TX) at a frequency of $2 \mathrm{~Hz}$ and a temperature of $22^{\circ} \mathrm{C}$, for shear strains from 0.1 to 10 , in a cylindrical tube with a diameter of $1 \mathrm{~mm}$. The sample was allowed to rest for 30 seconds after being drawn into the measurement tube, to allow the internal blood structures to reach a quiescent state.

Statistical analysis was performed using XLSTAT 7.5.2 (Addinsoft, New York, NY). Pearson correlations were performed

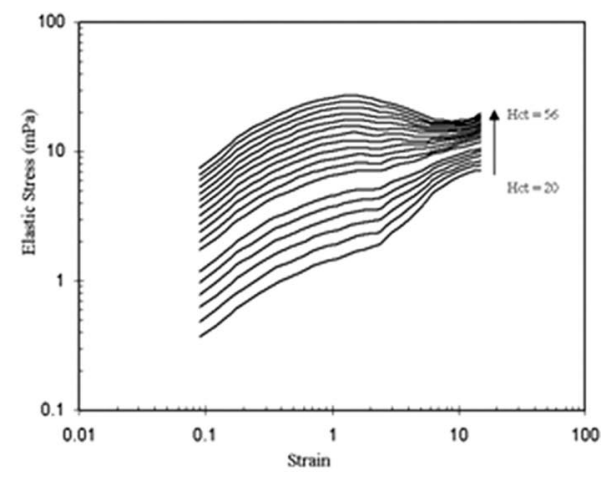

Figure 2. The relaxation time (left) and elastic stress (right) of pediatric blood for $20 \%$ to $56 \%$ hematocrits, in increments of $2 \%$. 


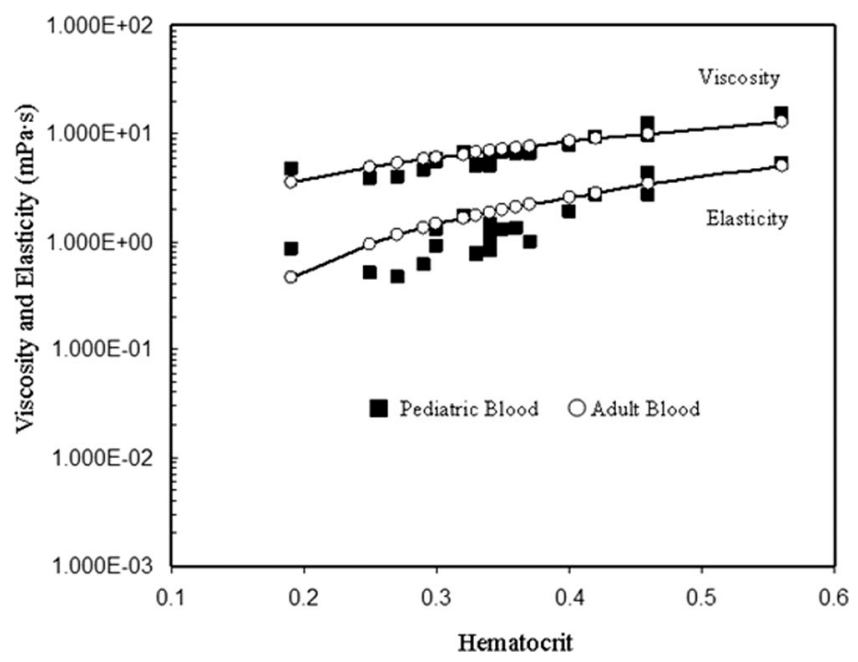

Figure 3. Pediatric viscoelasticity data compared with reference adult data at a strain of 0.2 .

between age, hematocrit, and viscoelastic parameters. Nonlinear regression equations were developed for viscosity and elasticity as functions of hematocrit at strains of 0.2 (quiescent aggregated state), 1 (the ultimate breakup of the aggregates), and 5 (cell deformation and layer formation). ${ }^{9}$ A Student's $t$ test was also used to compare pediatric blood data to reference adult data. ${ }^{10}$ Values for $p<0.05$ were considered statistically significant.

\section{Results}

The mean patient age was $20.4 \pm 30.0$ months, ranging from 4 days to 7.7 years. The mean hematocrit was $35.6 \% \pm$ $7.2 \%$. The results for viscosity and elasticity from a shear rate of 1.0-200 s $\mathrm{s}^{-1}$ are shown in Figure $\mathbf{1}$ for blood at three hematocrit levels. Aqueous xanthan gum/glycerin/sodium iodide solutions were used as blood analogs. A solution of 0.03 weight percent xanthan gum, 16\% glycerin, and 50\% sodium iodide was used to model pediatric blood at a $40 \%$ hematocrit.

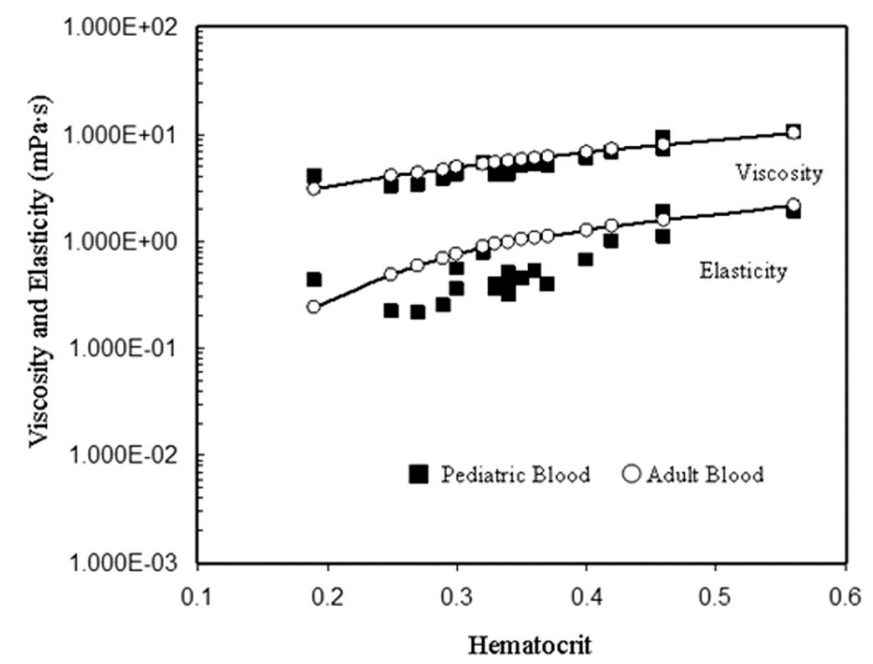

Figure 4. Pediatric viscoelasticity data compared with reference adult data at a strain of 1 .

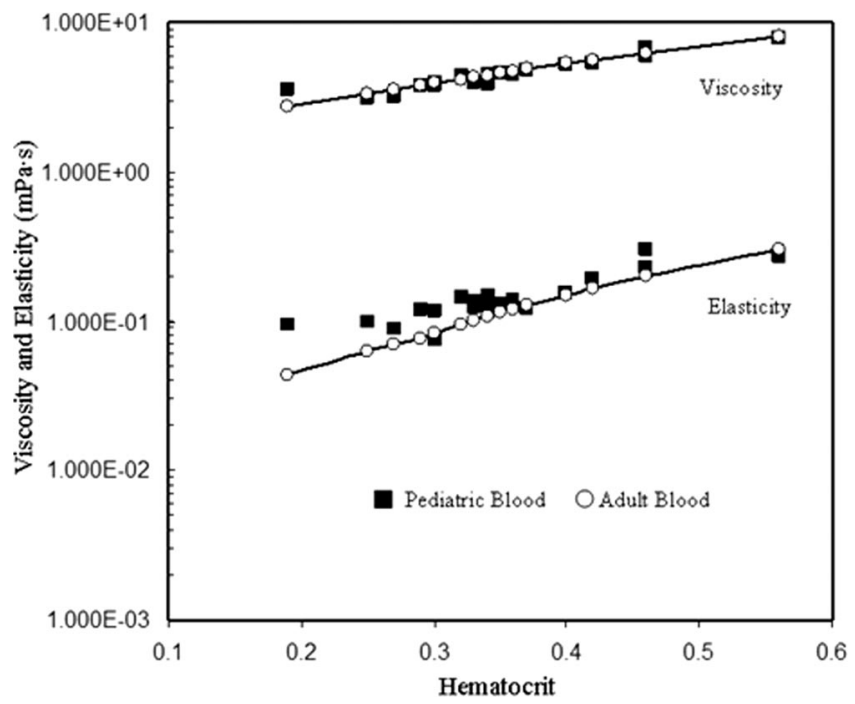

Figure 5. Pediatric viscoelasticity data compared with reference adult data at a strain of 5 .

Similarly, a solution of $0.009 \%$ xanthan gum, $15 \%$ glycerin, and $50 \%$ sodium iodide was used to model pediatric blood at a $20 \%$ hematocrit, and $0.05 \%$ xanthan gum, 22\% glycerin, and $50 \%$ sodium iodide was used to model pediatric blood at a $60 \%$ hematocrit. The sodium iodide was added to increase the refractive index of the solution in order to match the refractive index of the acrylic models that we use to perform flow visualization experiments. It does not affect the viscoelasticity of the analog solution.

The relaxation time and elastic stress are shown in Figure 2. The curves are plotted for hematocrit values of $20 \%$ to $56 \%$, in increments of $2 \%$. The yield stress is characterized by the maximum stress, and occurs at a strain of approximately 1. Hematocrits $<40 \%$ are not characterized by a maximum stress; hence, they have no elastic yield stress.

\section{Age and Viscoelasticity}

There was no correlation between patient age and viscosity at any of the strain values. There was a slight correlation between patient age and elasticity at a strain of $1(0.554, p=$ $0.011)$. There was no correlation at the other values of strain.

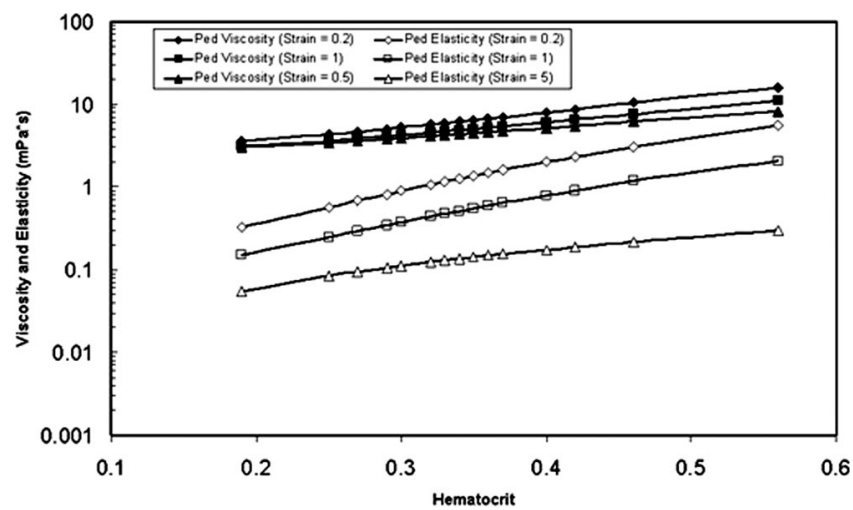

Figure 6. Regression equations for pediatric viscosity and elasticity as a function of hematocrit at strains of $0.2,1$, and 5 . 
Table 1. Viscoelasticity Equations at Strains of $0.2,1$, and 5

\begin{tabular}{|c|c|c|c|c|}
\hline \multirow[b]{2}{*}{ Strain $=0.2$} & \multicolumn{2}{|c|}{ Pediatric blood $(\mathrm{mPa} \cdot \mathrm{s})$} & \multicolumn{2}{|c|}{ Adult blood (mPa $\cdot \mathrm{s})$} \\
\hline & $\begin{array}{l}\eta^{\prime}=2.96+64.73 \mathrm{H}^{2.80} \\
\eta^{\prime \prime}=0.15+33.75 \mathrm{H}^{3.17}\end{array}$ & $\begin{array}{l}R^{2}=0.915 \\
R^{2}=0.871\end{array}$ & $\begin{array}{l}\eta^{\prime}=0.3+25.47 \mathrm{H}^{1.252} \\
\eta^{\prime \prime}=34.84 \mathrm{H}^{2.6013} \\
\eta^{\prime \prime}=15.9 \mathrm{H}^{1.997}\end{array}$ & $\begin{array}{l}\mathrm{H}=0.15 \text { to } 0.50 \\
\mathrm{H}=0.15 \text { to } 0.28 \\
\mathrm{H}=0.29 \text { to } 0.50\end{array}$ \\
\hline Strain $=1$ & $\begin{array}{l}\eta^{\prime}=2.64+38.88 \mathrm{H}^{2.66} \\
\eta^{\prime \prime}=0.08+11.95 \mathrm{H}^{3.08}\end{array}$ & $\begin{array}{l}\mathrm{R}^{2}=0.906 \\
\mathrm{R}^{2}=0.789\end{array}$ & $\begin{array}{l}\eta^{\prime}=1.27+21.3 \mathrm{H}^{1.4796} \\
\eta^{\prime \prime}=14.75 \mathrm{H}^{2.4693} \\
\eta^{\prime \prime}=5.39 \mathrm{H}^{1.579}\end{array}$ & $\begin{array}{l}\mathrm{H}=0.15 \text { to } 0.50 \\
\mathrm{H}=0.15 \text { to } 0.30 \\
\mathrm{H}=0.31 \text { to } 0.50\end{array}$ \\
\hline Strain $=5$ & $\begin{array}{l}\eta^{\prime}=2.58+22.10 \mathrm{H}^{2.36} \\
\eta^{\prime \prime}=0.73 \mathrm{H}^{1.56}\end{array}$ & $\begin{array}{l}R^{2}=0.936 \\
R^{2}=0.787\end{array}$ & $\begin{array}{l}\eta^{\prime}=1.73+17.1 \mathrm{H}^{1.709} \\
\eta^{\prime \prime}=0.399 \mathrm{H}^{1.3328} \\
\eta^{\prime \prime}=9.98 \mathrm{H}^{2.0662}\end{array}$ & $\begin{array}{l}\mathrm{H}=0.15 \text { to } 0.50 \\
\mathrm{H}=0.15 \text { to } 0.29 \\
\mathrm{H}=0.30 \text { to } 0.50\end{array}$ \\
\hline
\end{tabular}

There was a correlation between patient age and relaxation time at a strain of $1(0.608, p=0.004)$. There was no correlation at the other values of strain.

\section{Comparison of Pediatric to Adult Blood}

When comparing pediatric to adult blood reference values, the differences between the viscosities were not significant. The only significant difference between pediatric and adult elasticity was found at a strain of $0.2(p=0.007)$. Pediatric viscoelasticity values are shown with adult reference data ${ }^{10}$ in Figures 3 to 5 , at strains of $0.2,1$, and 5, respectively. The regression equations for viscosity and elasticity as a function of hematocrit are shown in Table $\mathbf{1}$ along with reference equations developed by Thurston et al. ${ }^{10}$ The equations are also shown in Figure 6, with the viscosity and elasticity shown at as a function of hematocrit at three values of strain.

\section{Discussion}

As expected, there is a strong dependence on the hematocrit viscoelastic parameters. The hematocrit levels for pediatric patients are lower than that considered normal for adult patients, and the variability of viscoelasticity due to hematocrit must be taken into account. This dependence on hematocrit should be taken into consideration when developing blood analogs.

The relaxation time varies greatly with shear rate and hematocrit as does the rouleaux size. ${ }^{11}$ This complicates modeling the shear dependent behavior of pediatric blood, and indicates (as is well known) that the single Maxwell element is not an appropriate model for (pediatric) blood. A successful model may have multiple "relaxation" times, all independent of the applied shear rate.

The relative importance of the relaxation time to the flow is characterized by the Deborah number $\left(\mathrm{N}_{\mathrm{DE}}\right){ }^{8}$ It is the relationship of relaxation time to the characteristic time scale of the fluid $\left(\mathrm{t}_{\mathrm{e}}\right)$ :

$$
\mathrm{N}_{\mathrm{DE}}=\frac{\lambda}{t_{\mathrm{e}}}
$$

For a $\mathrm{N}_{\mathrm{DE}} \approx 1$ the material is viscoelastic, for $\mathrm{N}_{\mathrm{DE}}<<1$ the dissipative effects of viscosity dominate, for $N_{D E}>>1$ elasticity dominates. For blood, the $\mathrm{N}_{\mathrm{DE}}$ can be considered to be an index of aggregation tendencies blood based on the flow characteristics. The effect of hematocrit on the aggregation tendency of red blood cells is evident upon examination of the elastic stress curves. The lower hematocrit levels exhibit a lower stress overall and do not display a maximum stress value through a transition to high strain conditions.

\section{Conclusion}

We found no correlation between patient age and blood viscoelasticity. There was also no statistical significance between pediatric viscosity and adult viscosity. There were only minor differences between adult and pediatric blood, which allows the same blood analog to be used in both cases. The composition of analog fluids for $20 \%, 40 \%$, and $60 \%$ hematocrits are given as functions of xanthan gum, glycerin, and sodium iodide solution. The sodium iodide is added solely to provide an index of refraction match to acrylic. Because the nature of the viscoelasticity, particularly the elastic component, changes with hematocrit, care should be taken in interpreting flow data from models employing blood analog solutions.

\section{Acknowledgments}

The authors would like to thank George Thurston and Nancy Henderson for their assistance in making the viscoelasticity measurements. This study was supported by NIH NHLBI Contract HV 48191.

\section{References}

1. Cohen G, Permut L: Decision making for mechanical cardiac assist in pediatric cardiac surgery. Pediatr Card Surg Ann 8: 41-50, 2005.

2. Bachmann C, Hugo G, Rosenburg G, et al: Fluid dynamics of a pediatric ventricular assist device. Artif Organs 24: 362-372, 2000.

3. Stuart J, Kenny MW: Blood rheology. J Clin Pathol 33: 417-429, 1980.

4. Kasser U, Heimburg P, Walitza E: Viscoelasticity of whole blood and its dependence on laboratory parameters. Clin Hemeoreol 9: 307-312, 1989.

5. Thurston GB: Effects of hematocrit on blood viscoelasticity and in establishing normal values. Biorheology 15: 239-249, 1978.

6. Walitza E, Anadere H, Chmiel H, Witte S: Evaluation of viscoelasticity measurements of human blood. Biorheology 25: 209217, 1988.

7. Thurston GB: The elastic yield stress of human blood. Biomed Sci Instrum 29: 87-93, 1993.

8. Darby R: Viscoelastic Fluids: An Introduction to Their Properties and Behavior. New York, Marcel Dekker, 1976.

9. Thurston GB : Rheological parameters for the viscosity, viscoelasticity and thixotropy of blood. Biorheology 16: 149-162, 1979.

10. Thurston GB, Henderson NM, Jeng M: Effects of erythrocytapheresis transfusion on the viscoelasticity of sickle cell blood. Clin Hemeoreol 30: 83-97, 2004.

11. Anand M, Rajagopal KR: A shear-thinning viscoelastic fluid model for describing the flow of blood. Int J Cardiol Med Sci 4: 59-68, 2004. 